\title{
Gallium scanning by conventional imaging and emission computed tomography in the pretreatment evaluation of lung cancer
}

\author{
DL BROUGHTON, CJ GIBSON, T CRAKE, SJ PEARCE, RCF LEONARD \\ From the Department of Medicine and Regional Medical Physics, Dryburn Hospital, Durham, and the \\ Department of Clinical Oncology, University of Edinburgh
}

ABSTRACT Gallium 67 citrate was evaluated with conventional scanning and emission computed tomography (CT) scanning as a method of pretreatment staging of the intrathoracic, especially mediastinal, spread of lung cancer. Of 31 patients with tumours of various histological types, the isotope was concentrated in the primary lesion in all but one. In 10 out of 12 patients who underwent surgical exploration conventional gallium scanning correctly indicated the mediastinum to be clear and identified two other patients with a tumour of the mediastinum not recognised by chest radiography or emission CT gallium scanning. Neither conventional nor emission CT gallium scanning produced false positive images. Conventional gallium scanning can give information about the mediastinum not available from chest radiographs or bronchoscopy.

Since the introduction of gallium 67 citrate into clinical medicine by Edwards and Hays in $1969^{1}$ there have been several studies of its use in lung cancer. These have shown a sensitivity for detecting the primary lesion of $50-100 \%$, as well as variation in its accuracy in assessing the mediastinum..$^{2-6}$ Most of the studies were performed with a rectilinear scanner. Since the introduction of improved imaging equipment, including the gamma camera, and facilities for performing emission computed tomography (CT) there have been only a few studies examining the effect of these advances on the accuracy of gallium imaging in lung cancer.?

Our aim was to see whether the improvements could increase the sensitivity of gallium 67 imaging for the primary lesion, and also increase its sensitivity and specificity in the detection of mediastinal node tumours.

\section{Methods}

Thirty one consecutive patients were assessed in a coded prospective study. They presented with clini-

Address for reprint requests: Dr RCF Leonard, Department of Clinical Oncology, Western General Hospital, Edinburgh EH4 2XU.

Accepted 5 September 1984 cal and radiological evidence of lung cancer, which was subsequently confirmed in 28 cases histologically and in three cases by the clinical and radiological course of the disease.

After bronchoscopy each patient was given 160 $\mathrm{MBq}$ of gallium 67 citrate and had conventional (Ga-C) and emission CT images recorded of the thorax 72 hours after injection. Imaging was performed with a large field of view gamma camera (IGE 400T) that had a medium energy collimator and a triple peak energy window. For the emission CT sequence a series of 64 views was obtained at equiangular intervals with an acquisition time of 20 seconds per view. Transverse sections through the thorax were reconstructed from this series of projection images.

The conventional gallium images, emission CT data, and chest radiographs were assessed separately by a clinician (RCFL) and a physicist (CJG). For the interpretation of the emission CT data two techniques were used. The original series of 64 views was displayed as a rapid movie, giving the appearance of the patient rotating in front of the camera, with good depth perception from the motion parallax effect. ${ }^{8}$ The reconstructed transverse sections were also viewed in the conventional manner. Representative views from an emission $C T$ sequence are shown in figure 1 . The reconstructed transverse views were also viewed in the conventional manner. Figure 


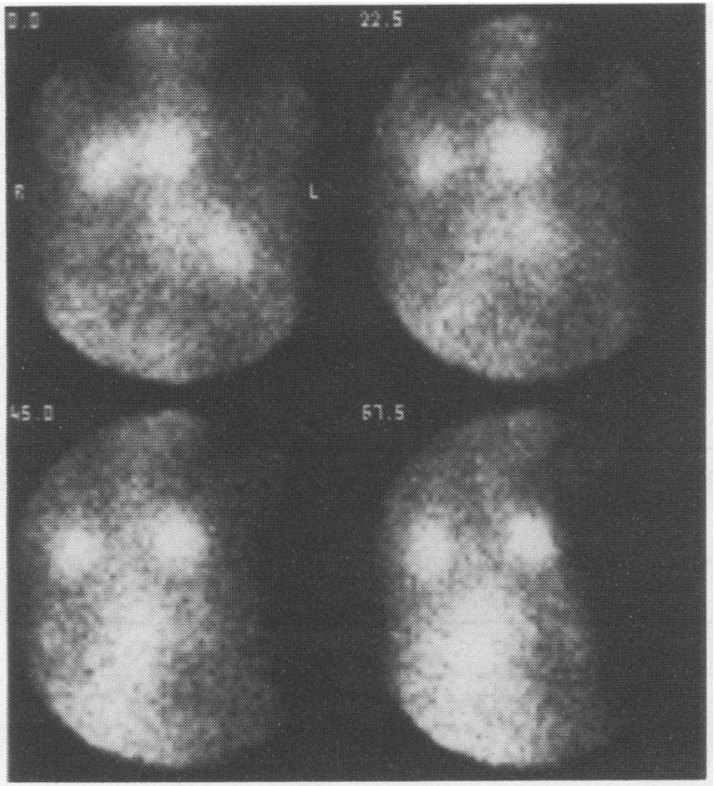

Fig 1 Four views from an emission computed tomography sequence at $0^{\circ}, 22.5^{\circ}, 45^{\circ}$, and $67.5^{\circ}$ of rotation towards the right lateral aspect. The changing positions of the three areas of uptake indicate their locations within the thorax. When viewed as a rapid movie sequence the relative depths of the lesions are perceived directly.

shows transverse sections through the thorax of the patient shown in figure 1. Further three dimensional information may be obtained by transforming the transverse sections into other planes. The additional information obtained by taking a series of views may increase sensitivity and may provide a more complete description of the position of the lesion. This is shown in figure 4 .

\section{Results}

The effectiveness of the different methods of gallium scanning in detecting primary tumours of different cell type is shown in table 1 .

Twelve patients subsequently had a thoracotomy

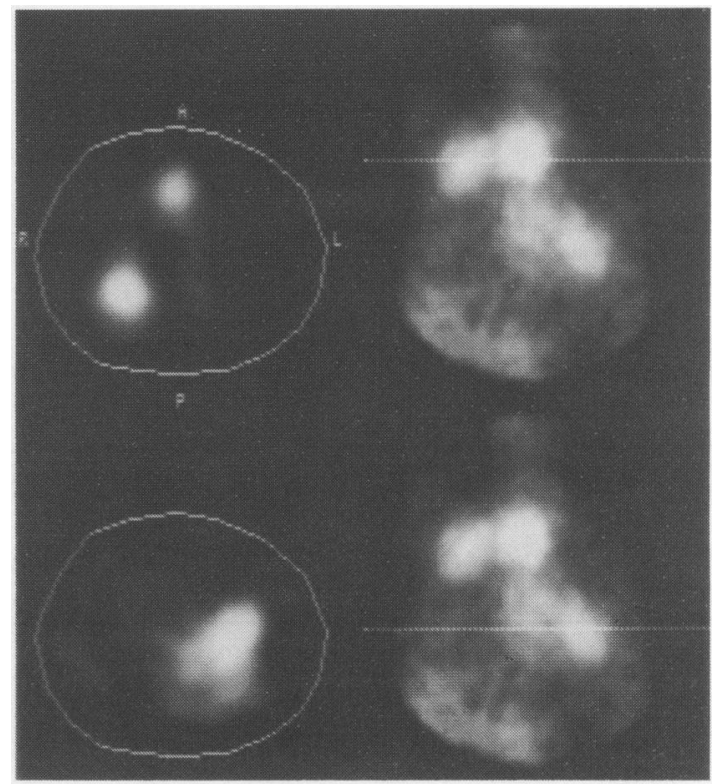

Fig 2 Transverse sections through the thorax (same patient as fig 1) at the levels indicated. Approximate body outlines are shown to aid interpretation. The lesion in the left lung is shown by the transverse sections to be both large and irregular, information that is not readily perceived with conventional imaging.

and the relationship between the results of gallium imaging and histological examination of the mediastinal and hilar nodes is shown in table 2. Two patients had disease in the mediastinal nodes; both had been detected by $\mathrm{Ga}-\mathrm{C}$ (one positive and one equivocal), whereas the chest radiographs had been equivocal and negative respectively. Both emission CT reconstructions however, gave negative results. In the 10 patients who had disease free mediastial nodes at surgery all had been judged to be free from mediastinal lesions by $\mathrm{Ga}-\mathrm{C}$, but two patients had equivocal chest radiographs. The emission CT reconstructions agreed with conventional imaging except in one case, in which the rotating image was equivocal. Two patients in the disease free group did

Table 1 Detection of the primary tumour with gallium 67 imaging

\begin{tabular}{lccc}
\hline Cell type of carcinoma & \multicolumn{2}{l}{ No of primary tumours detected/No of patients scanned } \\
\cline { 2 - 3 } & Conventional & Emission CT (rotation) \\
\hline Squamous cell & $16 / 16$ & $14 / 14$ & Emission CT (slice) \\
Adenocarcinoma & $5 / 6$ & $4 / 4$ & $14 / 14$ \\
Large cell & $3 / 3$ & $2 / 2$ & $3 / 4$ \\
Small cell & $3 / 3$ & $3 / 3$ & $2 / 2$ \\
Unclassified & $3 / 3$ & $3 / 3$ & $3 / 3$ \\
Total & $30 / 31$ & $26 / 26$ & $25 / 26$ \\
\hline
\end{tabular}


Table 2 The detection of mediastinal disease by chest radiography and different gallium 67 imaging modes in patients undergoing surgery

\begin{tabular}{|c|c|c|c|c|}
\hline \multirow[t]{3}{*}{ Imaging results } & \multicolumn{4}{|l|}{ Imaging modality } \\
\hline & \multirow[t]{2}{*}{ Chest radiograph } & \multicolumn{3}{|l|}{ Gallium 67} \\
\hline & & Conventional & Rotation & Slice \\
\hline \multicolumn{5}{|c|}{ Disease present 2 patients } \\
\hline Positive & 0 & 1 & 0 & 0 \\
\hline Equivocal & 1 & 1 & 0 & 0 \\
\hline Negative & 1 & $\mathbf{0}$ & 2 & 2 \\
\hline \multicolumn{5}{|c|}{ Disease absent 10 patients ${ }^{*}$} \\
\hline Positive & 0 & 0 & $\mathbf{0}$ & 0 \\
\hline Equivocal & 2 & 0 & 1 & $\mathbf{0}$ \\
\hline Negative & 8 & 10 & 7 & 8 \\
\hline
\end{tabular}

*Only eight of the disease free group had emission computed tomography.

Table 3 Detection of mediastinal disease by bronchoscopy, chest radiography, and gallium 67 scanning in non-surgical patients

\begin{tabular}{llll}
\hline Investigative results & Bronchoscopy & Chest radiography & Conventional $^{67}$ Ga \\
\hline $\begin{array}{l}\text { High probability of mediastinal } \\
\text { disease (13 patients) }\end{array}$ & & & 12 \\
$\quad \begin{array}{l}\text { Positive } \\
\text { Equivocal }\end{array}$ & 10 & 8 & 1 \\
$\begin{array}{l}\text { Negative } \\
\text { liw probability of mediastinal }\end{array}$ & 0 & 0 & 0 \\
$\begin{array}{l}\text { disease (6 patients) } \\
\quad \text { Positive }\end{array}$ & 0 & 5 & 3 \\
$\quad$ Equivocal & 0 & 0 & 0 \\
$\quad$ Negative & 6 & 0 & 3 \\
\hline
\end{tabular}

not have emission CT imaging performed. The patient with the equivocal rotation image had an adenocarcinoma and in this case the primary tumour was not evident from conventional imaging and only an equivocal finding on emission CT rotation views.

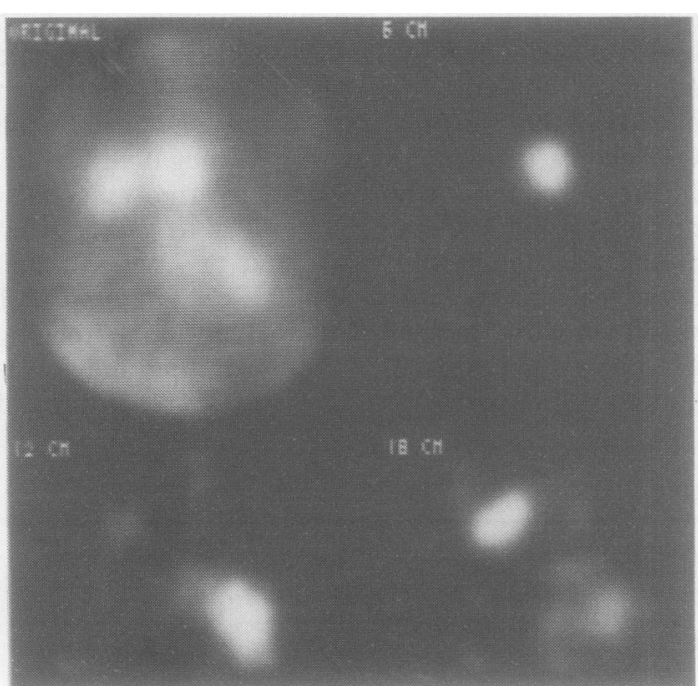

Fig 3 Conventional gallium image with coronal sections at 6,12 , and $18 \mathrm{~cm}$. The increased contrast in the section images helps to define the positions of the lesions.

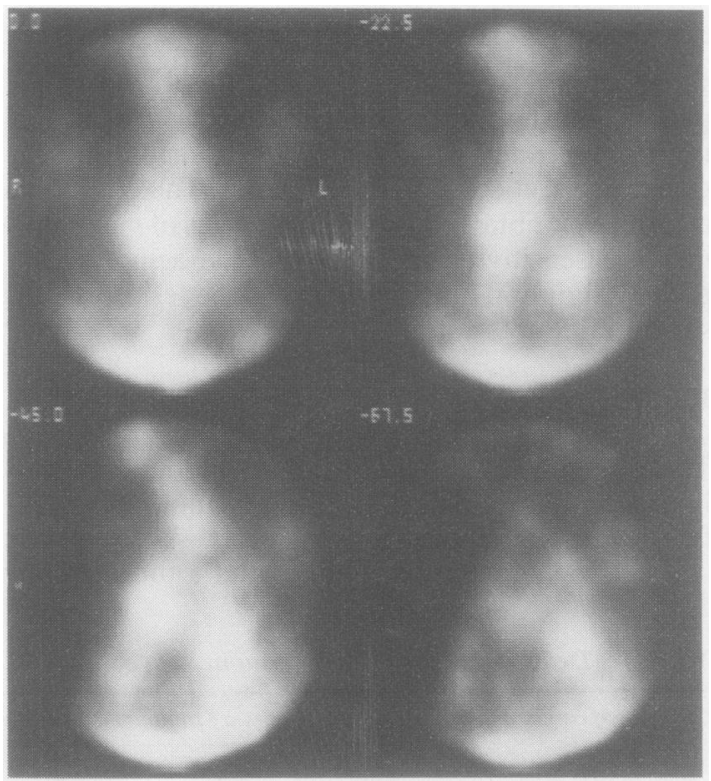

Fig 4 Four views from an emission computed tomography sequence at $0^{\circ}, 22 \cdot 5^{\circ}, 45^{\circ}$, and $67 \cdot 5^{\circ}$ of rotation towards the left lateral aspect. In the anterior view a suspicious area is seen in the medial part of the middle zone of the left lung. On the oblique views this is shown clearly as a large lesion in the substance of the left lung. 
Thus conventional imaging was both sensitive and specific for mediastinal disease whereas emission CT did not detect the disease in the two node positive patients. It was also less specific in that there was one equivocal result for mediastinal disease when the mediastinum was negative histologically.

The remaining 19 patients were divided into two groups according to their probability of having mediastinal disease. The high probability group had positive evidence of mediastinal spread from chest radiography or bronchoscopy or both (paralysed vocal cord, widened carina, bulging and distorted trachea or main bronchi). The low probability group lacked all of these features. The results of gallium imaging in these groups are shown in table 3. Of 13 patients with a high probability of mediastinal disease, 12 had a positive and one an equivocal image with $\mathrm{Ga}-\mathrm{C}$ of the mediastinum. Ten of the $13 \mathrm{had}$ emission CT performed, and all of these showed evidence of mediastinal disease. Thus conventional gallium imaging displayed mediastinal disease when this was also visible from the use of chest radiography and bronchoscopy together; it appears to be more sensitive than either chest radiography or bronchoscopy alone. ${ }^{4}$ In the low probability group there were discordant results and we cannot make any valid statement about this group. These six patients did not undergo thoracotomy-two refused operation, two were unsuitable because of poor lung function, one had intrabronchial extension affecting the carina, and one had small cell carcinoma.

Although overall the computed tomographic images did not add to the performance of conventional gallium imaging there were cases where we were impressed by the ability of the tomographic images to give additional information about the size, shape, and location of abnormal features. Examples are shown in figures $2-4$.

\section{Discussion}

We found conventional gallium imaging to be a very sensitive technique for the detection of the primary lesion in all histopathological subgroups. It was also accurate in the assessment of mediastinal disease in patients who could be evaluated, giving a positive or equivocal mediastinal image when disease was present in mediastinal nodes biopsied surgically and a negative image in all the patients with histologically normal mediastinal nodes. Emission computed tomography did not improve the detection rate and in the assessment of the mediastinum it was less accurate, yielding false negative images in the two patients with diseased mediastinal nodes.

The detection rate for the primary lesion in our series is in keeping with that in other recent studies $^{35}$ and it is now generally agreed that gallium imaging has a sensitivity of the order of $90 \%$ for primary lesions of all histopathological types. ${ }^{7}$ Only one primary lesion, an adenocarcinoma, was not detected by conventional gallium imaging and this may have been related to its small size (it was judged to be $1.5 \times 2.5 \mathrm{~cm}$ radiographically). Other studies $^{356}$ have found that lesions around $1-2 \mathrm{~cm}$ in diameter or less are unlikely to be detected by this imaging technique. In addition, other workers ${ }^{3469}$ have shown a lower sensitivity, about $80 \%$, in the detection of primary adenocarcinomas than of other histopathological types.

One objection to the use of gallium in lung cancer is its lack of specificity. ${ }^{10}$ In our series two patients suspected of having lung cancer had equivocal primary lesions evident on the scans. Both of these were subsequently shown not to have lung cancer and so were excluded from the study. We do not consider gallium imaging useful as a diagnostic tool, though some workers have shown that it is helpful in some circumstances, especially in the assessment of circumscribed peripheral shadows. ${ }^{36}$ Another criticism is that adjacent infected, inflamed tissue ${ }^{10}$ takes up gallium and makes interpretation of the image difficult. In our series uptake of the gallium corresponded in all cases to the pattern of the tumour on the radiographs and was well delineated.

In the assessment of mediastinal disease we found conventional gallium imaging to be accurate, although in one of the patients with histologically diseased mediastinal nodes the mediastinal appearances were only "equivocal." In some studies there have been appreciable numbers of false positive $\mathrm{Ga}-\mathrm{C}$ scans $^{34}$ and in others some of the equivocal results were shown to be due to inflammatory changes in the nodes. ${ }^{56}$ Interestingly, in our series of 10 patients with tumour free mediastinal nodes two showed histological features of anthracosis, two reactive hyperplasia, and one chronic inflammatory changes. All had negative mediastinal appearances on conventional gallium imaging.

Negative mediastinal images accurately predicted tumour free mediastinal nodes in all cases. This finding has been noted by other workers in recent studies. $^{469}$ Consequently these workers recommended that patients with an equivocal or positive mediastinal image should have mediastinoscopy, but that those with a negative image could be spared mediastinoscopy before thoracotomy. Results from the patients in our study would support this policy although the number of $\mathrm{Ga}-\mathrm{C}$ positive patients is small.

Emission computed tomography was less accurate than conventional gallium scanning as mediastinal disease in two patients was not detected with emis- 
sion CT and there was also an equivocal result in one patient who had histologically normal mediastinal nodes. In the latter the primary lesion was also seen as equivocal on the emission CT rotation. It has been suggested ${ }^{4}{ }^{79}$ that if the primary lesion does not concentrate gallium then no valid statement can be made about the mediastinum. A possible explanation for the less accurate results obtained with emission CT is the reduced information density in the images. With an acquisition time of 20 seconds per view the signal to noise ratio in each image is reduced by a factor of 3 compared with conventional images (three minutes per view). When a series of rotation images is viewed as a rapid movie this effect is partially mitigated, since the observer integrates information over several images. When transverse sections are reconstructed, however, the noise amplification of the reconstruction process" produces images with a poor signal to noise ratio.

We conclude that although conventional gallium imaging may have a role for staging lung cancer, especially mediastinal disease, emission computed tomography is not a useful adjunct. Negative mediastinal appearances on a conventional gallium scan strongly suggest a normal mediastinum and an equivocal or positive scan may indicate the need for further investigation of the mediastinum, perhaps by mediastinoscopy, if surgery is being contemplated. It may become relevant in future to have more accurate information about the volume of inoperable tumour when assessing the results of non-surgical treatment such as radiotherapy or chemotherapy. It remains to be seen whether the three dimensional information provided by emission computed tomography can be used for this purpose.

\section{References}

1 Edwards CL, Hays RL. Tumour scanning with 67-gallium citrate. J Nucl Med 1969;10:103-5.

2 Johnston GS. Clinical applications of gallium in oncology. Int J Nucl Med Biol 1981;8:249-55.

3 Demeester JR, Bekerman C, Joseph JG, et al. Gallium-67 scanning for carcinoma of the lung. $J$ Thorac Cardiovasc Surg 1976;72:699-708.

4 Alazraki NP, Ramsdell JW, Taylor A, Friedman RJ, Peters RM, Tisi GM. Reliability of gallium scan, chest radiography to mediastinoscopy for evaluating mediastinal spread in lung cancer. Am Rev Respir Dis 1978; 117:415-20.

5 Pannier R, Verlinde I, Puspuidjono I, Willemot JP, De Pondt M. Value of gallium-67 scanning in the evaluation of mediastinal involvement in lung cancer. Acta Tuberc Pneumol Belg 1979;70:353-4.

6 Pannier R, Verlinde I, Puspuidjono I, Willemot JP. Role of gallium-67 thoracic scintigraphy in the diagnosis and staging of patients suspected of bronchial carcinoma. Thorax 1982;37:264-9.

7 Hoffer P. Status of gallium-67 in tumour detection. $J$ Nucl Med 1980;21:394-8.

8 Williams ED. Three dimensional radionuclide images. J Nucl Med 1981;22:193-4.

9 Peters RM. Staging of lung cancer. Chest 1977;71:633-4.

10 Gottschalk $Z$. Nuclear imaging in clinical oncology. Cancer 1981;47, suppl:1156-7.

11 Budinger TF. Physical attributes of single photon tomography. J Nucl Med 1980;21:579-92.

12 Gibson CJ, Leonard RCF, Aikenhead J. Evaluation of ECAT techniques for the measurement of Ga-67 uptake in the chest. $\mathrm{Br} J$ Cancer 1983;48:127 (abstract). 